\title{
Jugendliche in ihrer freien Zeit
}

\section{Ergebnisse der neuen Jugendstudie Baden-Württemberg}

MIRIAM SCHMID UND

WOLFGANG ANTES

Miriam Schmid ist Projektleiterin bei der Jugendstiftung BadenWürttemberg und dort u. a. zuständig für die Jugendstudie Baden-Württemberg.

Wolfgang Antes ist Geschäftsführer der Jugendstiftung BadenWürttemberg. Die Jugendstiftung Baden-Württemberg entwickelt, berät, fördert und verantwortet Jugendbildungsprojekte und Programmlinien, die an der Schnittstelle zwischen außerschulischer Jugendbildung, Schule und Berufsfeld wirken.

www.jugendstiftung.de

\author{
Das Feizeitverhalten junger Menschen hat sich unter \\ anderem durch neue Mediennutzung und die Ausweitung \\ von Ganztagsschulen stark verändert. Welche Bedeutung \\ in diesem Zusammenhang noch den Angeboten von \\ Kultureinrichtungen und von Vereinen zukommt, \\ untersuchte die neue Jugendstudie Baden-Württemberg.
}

Die Freizeit stellt einen Verhaltensraum dar, den Jugendliche nach ihren eigenen Vorstellungen gestalten können. Hier können sie Erlerntes in der Welt außerhalb der Schule umsetzen, sich und ihre Fähigkeiten ausprobieren, Bereiche abseits von Lehrplänen erkunden und dabei selbst bestimmen, welche Ziele sie mit ihren Aktivitäten erreichen möchten. Freizeit hat deshalb einen sehr hohen Stellenwert im Leben junger Menschen und übt eine Mehrfachfunktion als Sozialisations-, Moratoriums-, Distinktions- und Identifikationsfeld auf dem Weg des Erwachsenwerdens aus.

Die nach 2011 zweite repräsentative Jugendstudie Baden-Württemberg legte deshalb einen Fokus der Befragung auf das Thema »Freizeit". Weitere Themefelder waren Freundschaft, Geld, Medien, Schule, Engagement, Werte und Zukunft.

Die Studie ist ein gemeinsames Projekt der Jugendstiftung Baden-Württemberg und des Landesschülerbeirats, gefördert durch das Ministerium für Kultus, Jugend und Sport Baden-Württemberg. Gemeinsam mit Jugendlichen aus dem Vorstand des Landesschülerbeirats wurde der Fragebogen entwickelt, die Erhebungsmethoden festgelegt und die Durchführung der Befragung abgestimmt. Die Studie ist damit nicht nur eine statistische Erfassung von aktuellen Daten, sondern ein Jugendbildungs- und Beteiligungsprojekt. Besondere Bedeutung gewinnt neben den statistischen Darstellungen und Auswertungen die Kommentierung einzel- ner Teilergebnisse der Studie durch Wissenschaftler und Landesschülerbeirats.

Insgesamt wurden 2.396 Jugendliche zwischen 12 und 18 Jahren aus nahezu allen Stadt- und Landkreisen Baden-Württembergs klassenweise befragt. Dieser Stichprobe liegt die Übergangsquote von der Klassenstufe vier der Grundschule auf eine weiterführende Schulart zugrunde: 25 Prozent Hauptschüler, 34 Prozent Realschüler, 40 Prozent Gymnasiasten. Zusätzlich wurden Schüler und Schülerinnen einer Sonderschule evaluiert. Die befragten Jugendlichen wurden in drei Altersgruppen aufgeteilt: 12 bis 14,15 bis 16 und 17 bis 18 Jahre.

Die Jugendstiftung hat standardisierte Interviews mit Jugendlichen durchgeführt, in denen die Fragestellungen der Studie vertieft erörtert wurden.

\section{Ergebnisse Freizeit}

Die Möglichkeiten, wie Jugendliche ihre Freizeit gestalten können, werden immer zahlreicher und vielfältiger. Mit der Verbreitung von Handy, Tablet, Spielekonsole \& Co. sind in den letzten Jahren immer schneller immer neuere Angebote im medialen Bereich entstanden, die speziell auf junge Kunden zu- geschnitten sind. Aber auch Vereine, Museen und Jugendorganisationen haben erkannt, dass sie mehr tun müssen, um für Jugendliche attraktiv zu sein und neue Konzepte und Angebote geschaffen, mit denen sie junge Menschen für sich gewinnen 
wollen. Nicht zuletzt entwickeln auch die Schulen selbst, auf ihrem Weg zur Ganztagsbetreuung, verstärkt außerunterrichtliche Angebote für ihre Schülerinnen und Schüler.

Konkret interessierte uns, welche Freizeitangebote den Jugendlichen in ihrem Umfeld bekannt sind, wie sie diese bewerten und wie häufig sie von ihnen genutzt werden.

\section{Welche Freizeitmöglichkeiten vor Ort bekannt sind}

Die meisten Jugendlichen kennen in ihrer Wohnumgebung den Sportverein (89 Prozent) und öffentliche Sportplätze (86 Prozent). 72 Prozent der Befragten wissen, dass der Musikverein oder die Musikschule und die Bibliothek vor Ort einige Möglichkeiten bieten, um die freie Zeit zu gestalten. Deutlich mehr als die Hälfte kennt darüber hinaus den lokalen offenen Jugendtreff (66 Prozent) und die Freizeitangebote in der Schule (57 Prozent). Am wenigsten bekannt sind Jugendgruppen in Vereinen (49 Prozent) und Museen (41 Prozent).

\section{Welche Freizeitangebote intensiv genutzt werden}

Die Jugendlichen, die angeben, bestimmte Freizeitangebote zu nutzen, haben wir gefragt, wie oft sie dies tun.
Auf diesem Weg wollten wir herausfinden, welche Freizeitangebote intensiv genutzt werden. Intensivnutzer sind Jugendliche, die mehrmals oder mindestens einmal wöchentlich ein Angebot nutzen. Die Auswertung ergibt, dass der Sportverein (54 Prozent) gefolgt von den öffentlichen Sportplätzen (36 Prozent) am häufigsten genutzt wird. Schlusslichter bei dieser Betrachtungsweise sind die Bibliothek (11 Prozent) und Museen (2 Prozent).

\section{Welche Freizeitangebote seltener genutzt werden}

Berücksichtigt man neben den Intensivnutzern auch die Jugendlichen, die die einzelnen Freizeitangebote zwar seltener, aber dennoch mit gewisser Regelmäßigkeit nutzen, ergibt sich ein ausgeglicheneres Bild. Möglichkeiten, die öffentliche Sportplätze (67 Prozent) zur Freizeitgestaltung bieten, werden insgesamt von den Jugendlichen mehr genutzt als die in Sportvereinen (59 Prozent). Bibliotheken werden mit 57 Prozent von fast ebenso vielen der Befragten besucht wie der Sportverein, wenn auch von den meisten weniger oft. Bei den sonstigen Nennungen ergibt sich im Vergleich zur vorherigen Abbildung zur Intensivnutzung eine allgemeine Angleichung im Spektrum von 29 bis 39 Prozent.

\section{Welche Freizeitangebote von Mädchen, welche von Jungen intensiv genutzt werden}

Bei der Intensivnutzung von Freizeitangeboten interessierte uns, ob es geschlechtsspezifische Unterschiede gibt. Auffällig sind dabei die sichtbar werdenden Unterschiede bei vier Freizeitangeboten. Während Jungen deutlich häufiger im Sportverein (männlich 61 Prozent, weiblich 46 Prozent) und auf öffentlichen Sportplätzen (männlich 51 Prozent, weiblich 22 Prozent) anzutreffen sind, frequentieren Mädchen verstärkt die Angebote von Musikvereinen und Musikschulen (weiblich 32 Prozent, männlich 26 Prozent) und suchen häufiger Bibliotheken (weiblich14 Prozent, männlich 8 Prozent) auf.

\section{Welche Freizeitangebote intensiv nach Schularten genutzt werden}

Schulartübergreifend erhalten die Freizeitaktivitäten, die mit Sport in Verbindung stehen (Sportverein, öffentliche Sportplätze), durchschnittlich ebenfalls die höchsten Zustimmungsraten der Jugendlichen. Eine deutliche Tendenz ergibt sich bei der genaueren Betrachtung der Ergebnisse von Schülerinnen und Schülern der Haupt- und Werkrealschulen. Abgesehen von drei Ausnahmen (Sportverein, Musikverein und Musikschule, $\rightarrow$

Welche Freizeitmöglichkeiten kennst du in deinem Ort?

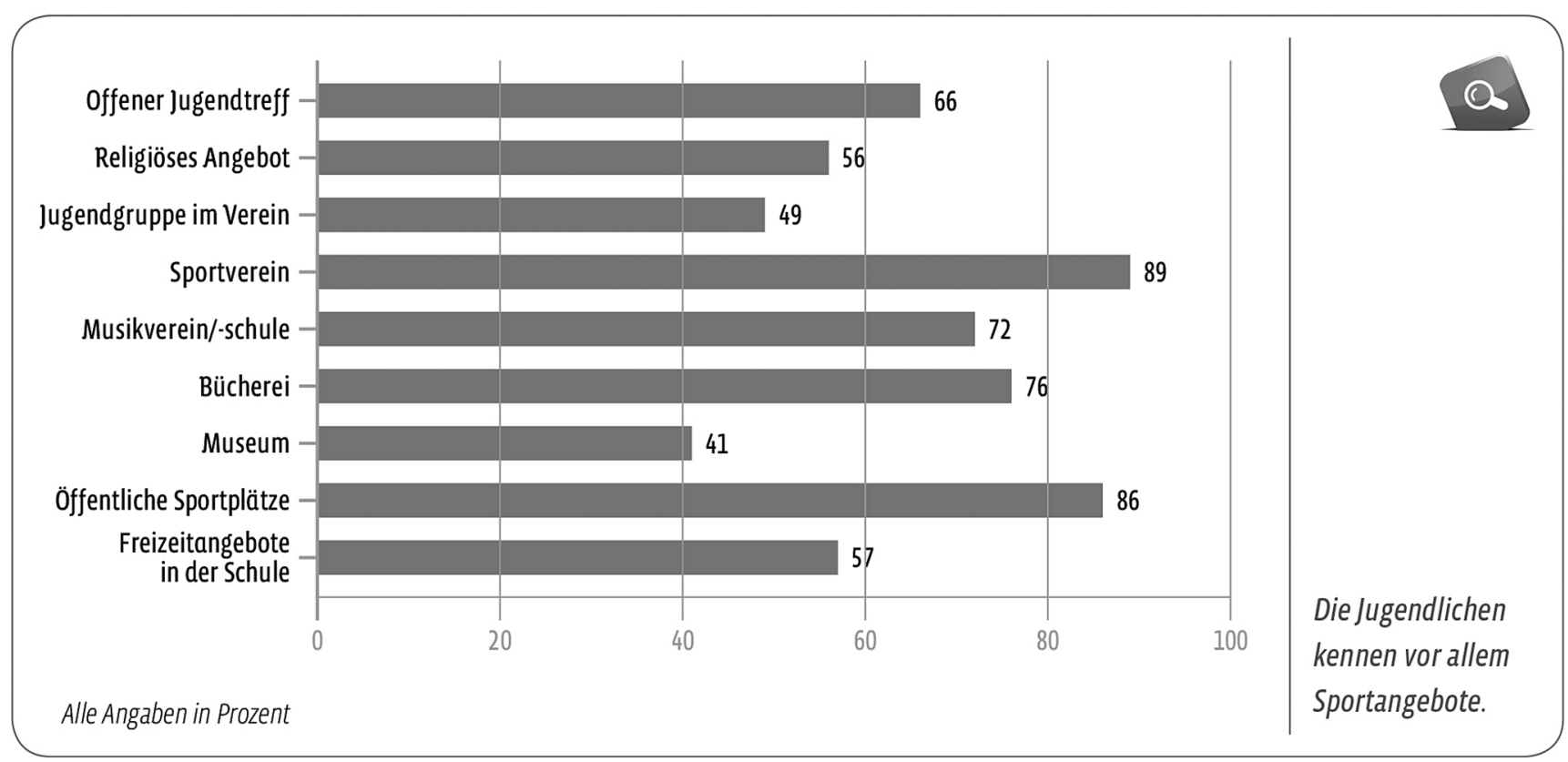


Welche Freizeitangebote nutzt du intensiv?

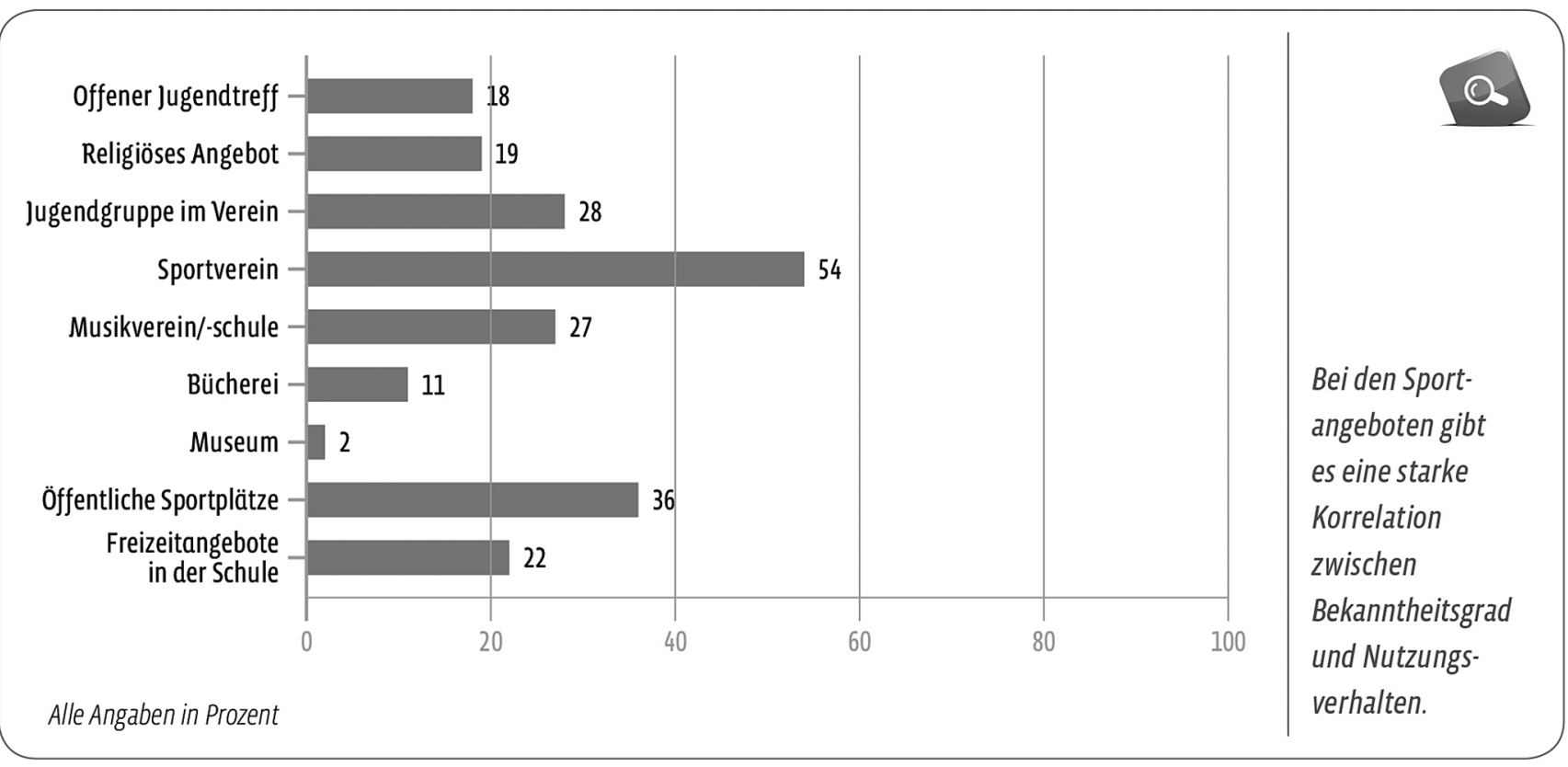

Welche Freizeitangebote nutzt du intensiv?

(Ergebnisse nach Geschlecht)

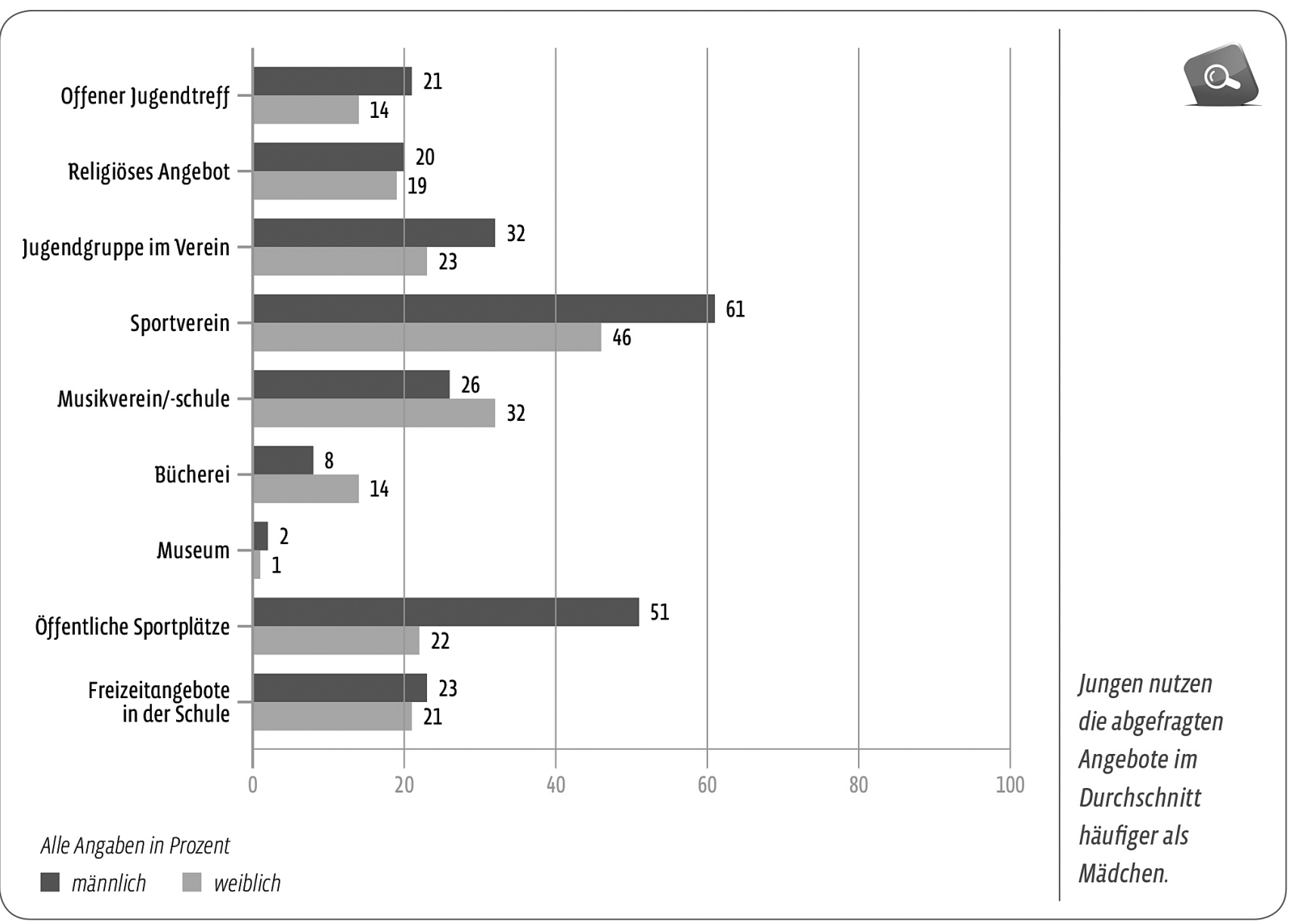


Welches Bild ergibt sich, wenn zusätzlich zu den Intensivnutzern auch diejenigen berücksichtigt werden, die die Freizeitangebote seltener nutzen?

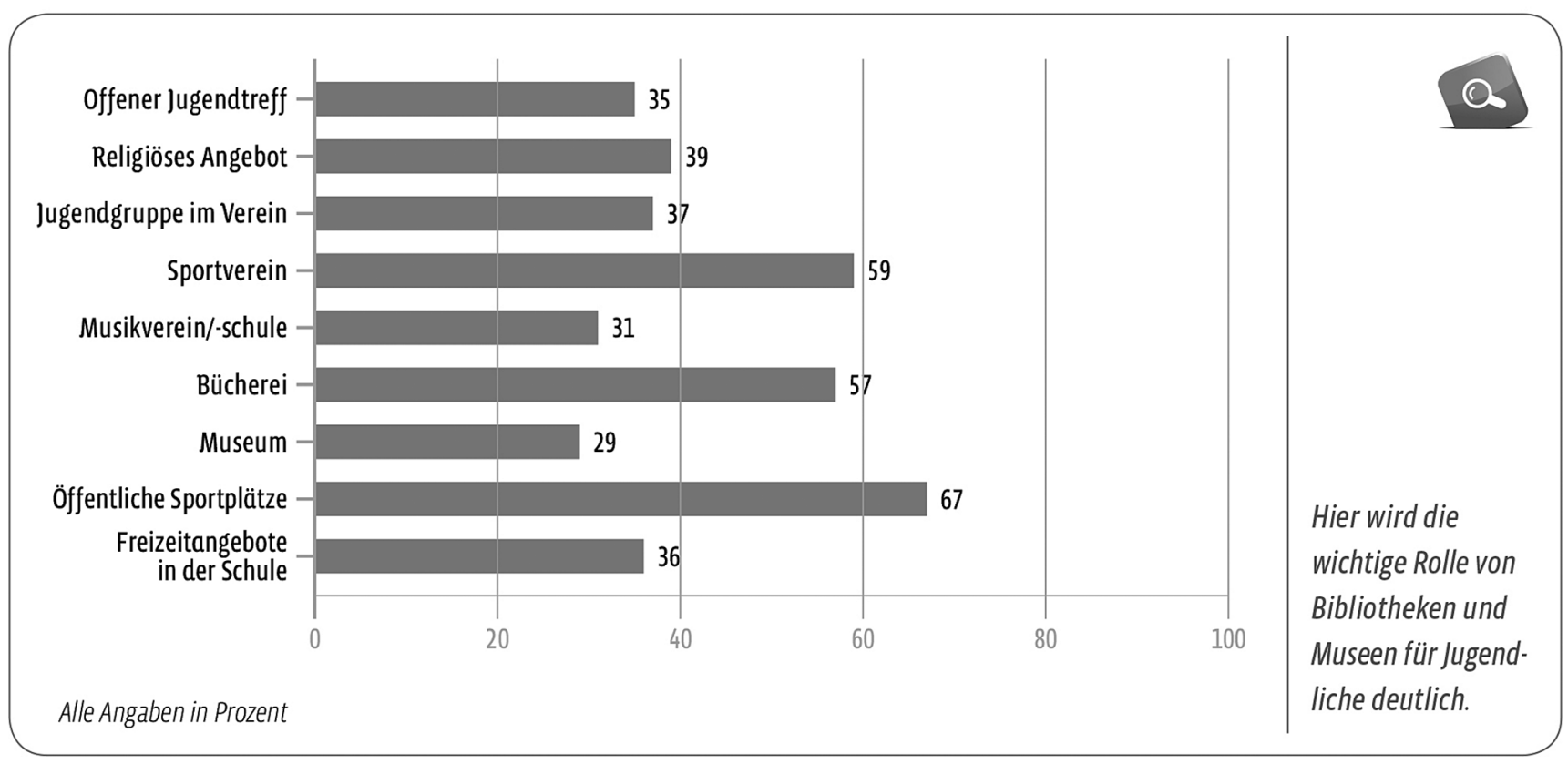

Welche Freizeitangebote nutzt du intensiv?

(Ergebnisse nach familiärer Herkunft)

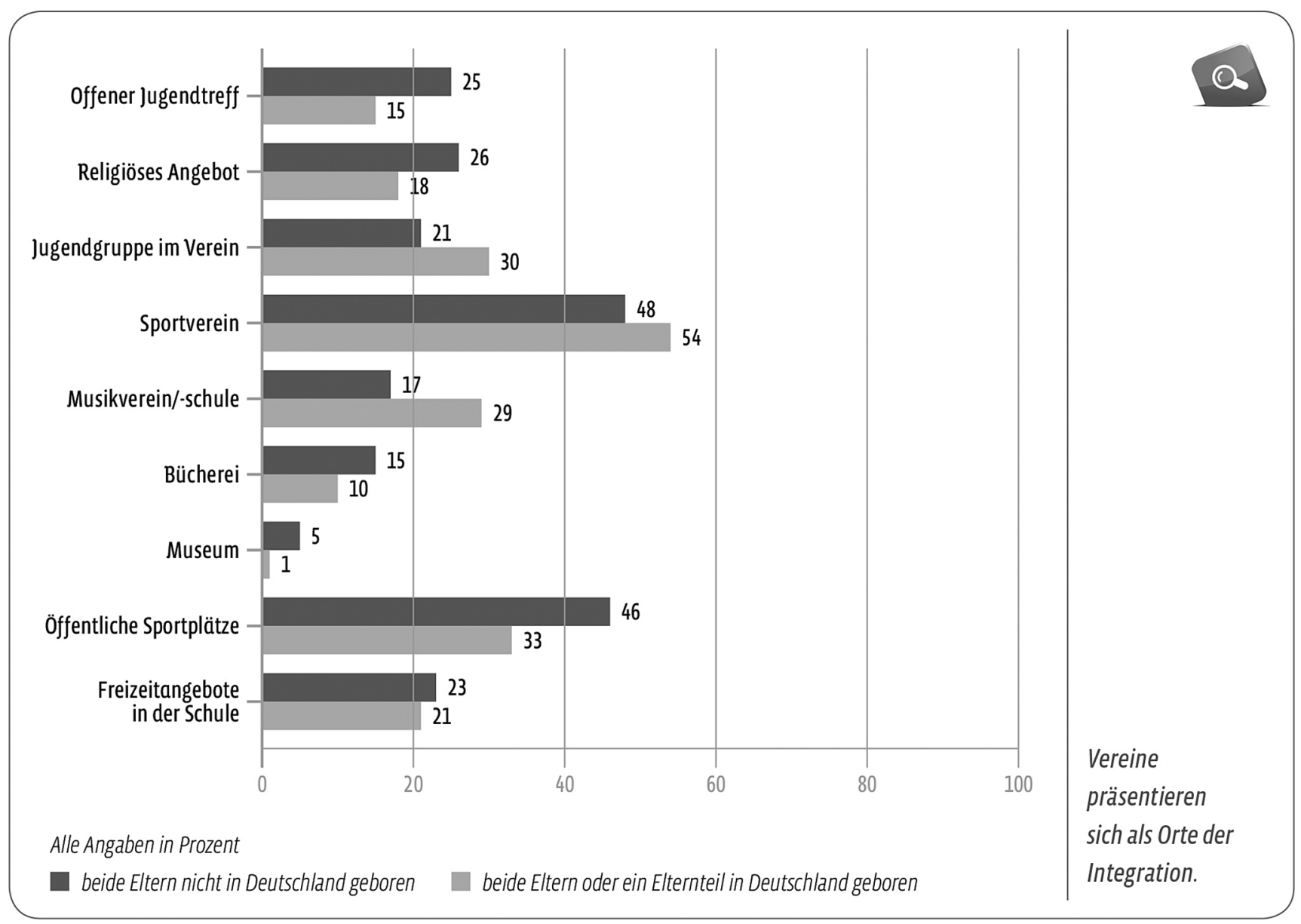


Welche Freizeitangebote nutzt du intensiv?

(Ergebnisse nach Schulart)

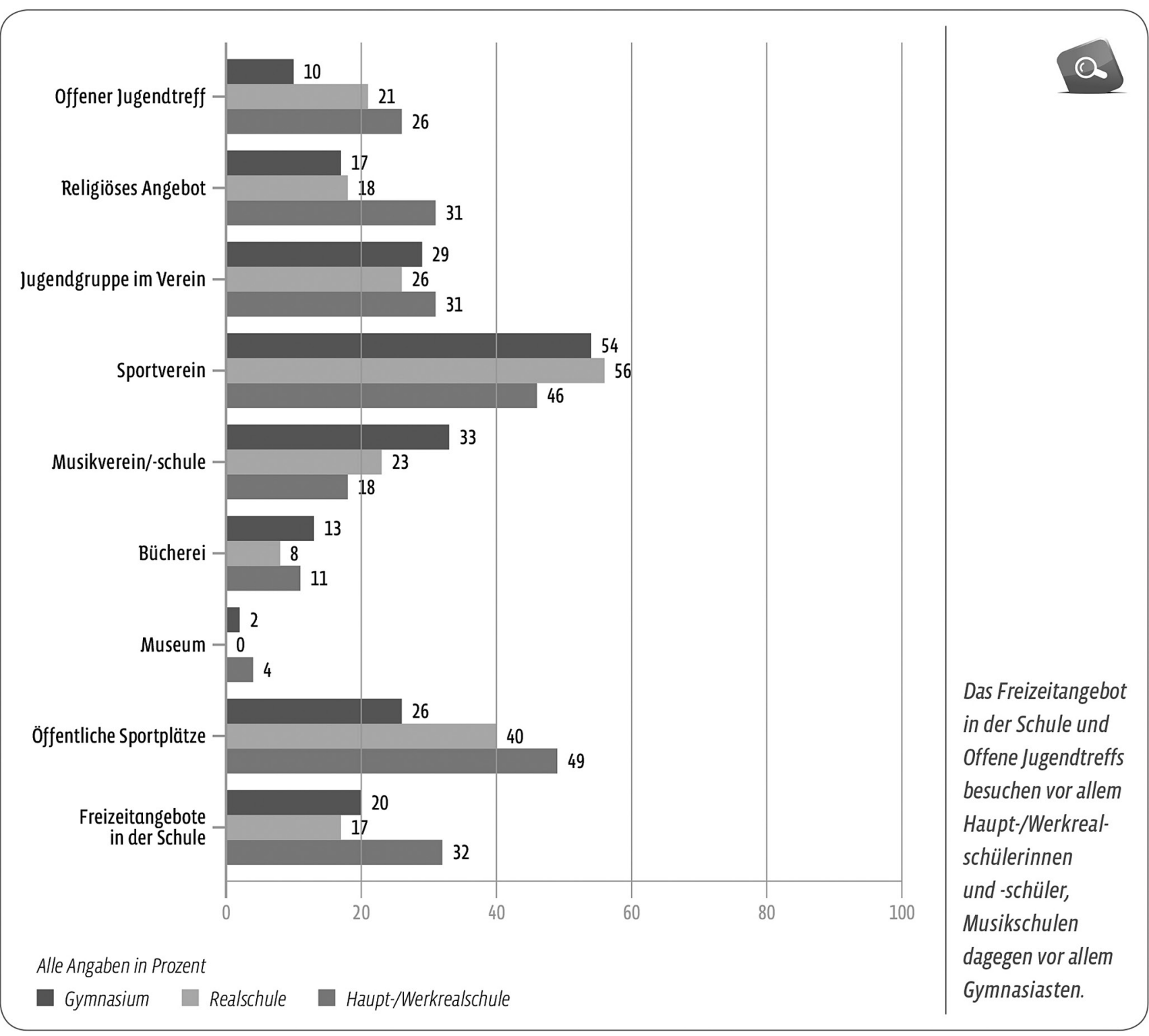

Bibliothek) zählen diese häufiger zu den Intensivnutzern bestehender Angebote vor Ort als Jugendliche der anderen Schularten. Besonders deutlich wird dies bei religiösen Freizeitbeschäftigungsmöglichkeiten oder auch bei Freizeitangeboten der Schule.

\section{Welche Freizeitangebote intensiv nach familiärer Herkunft genutzt werden}

Die Betrachtung der Intensivnutzer unter dem Aspekt der familiären Herkunft ergibt ein gemischtes Bild. Während Jugendliche, deren Eltern im Ausland geboren sind, häufiger die Angebote des offenen Jugendtreffs, religiöse Angebote, die Bibliothek und öffentliche Sportplätze nutzen, zeigen sich bei
Vereinsaktivitäten wahrnehmen, nicht gering. Die Vereine leisten somit einen wichtigen Beitrag zur Integration.

\section{"Die Jugendgruppenangebote von}

\section{Vereinen sind kaum bekannt»}

der Vergleichsgruppe andere Präferenzen. Kinder mit mindestens einem in Deutschland geborenen Elternteil sind häufiger in der Jugendgruppe eines Vereins (30 Prozent) und dem Sportverein (54 Prozent) oder Musikverein (29 Prozent) anzutreffen. Trotzdem ist die Zahl der Kinder aus Einwandererfamilien, die
Die Jugendstudie Baden-Württemberg 2013 erhalten Sie als PDF auf der Seite des Landesschülerbeirats www.lsbr.de oder unter www.jugendnetz.de.

Als Publikation können Sie die Jugendstudie auf www.jugendstiftung.de unter Publikationen für $18,00 €$ bestellen. 